\title{
Erratum to: Anosmin-1 over-expression increases adult neurogenesis in the subventricular zone and neuroblast migration to the olfactory bulb
}

\author{
Diego García-González ${ }^{1,6}$ - Verónica Murcia-Belmonte ${ }^{1,8} \cdot$ Pedro F. Esteban ${ }^{1}$ • \\ Felipe Ortega ${ }^{2}$ - David Díaz ${ }^{3}$ Irene Sánchez-Vera ${ }^{4,7} \cdot$ Rafael Lebrón-Galán $^{1}$. \\ Laura Escobar-Castañondo ${ }^{5}$ Luis Martínez-Millán ${ }^{5} \cdot$ Eduardo Weruaga $^{3}$. \\ José Manuel García-Verdugo $^{4} \cdot$ Benedikt Berninger $^{2} \cdot$ Fernando de Castro $^{1}$
}

Published online: 6 September 2016

(c) Springer-Verlag Berlin Heidelberg 2016

\section{Erratum to: Brain Struct Funct (2016)}

\section{1:239-260}

DOI 10.1007/s00429-014-0904-8

The original publication of this article failed to note that the article has two corresponding authors and not just one. The co-corresponding authors are Diego García-González (diego.garcia.gonzalez@gmail.com) and Fernando de Castro (fdecastro@cajal.csic.es).

The online version of the original article can be found under doi:10.1007/s00429-014-0904-8.

Diego García-González

diego.garcia.gonzalez@gmail.com

$\triangle$ Fernando de Castro

fdecastro@cajal.csic.es

1 Grupo de Neurobiología del Desarrollo-GNDe, Hospital Nacional de Parapléjicos, Toledo, Spain

2 University Medical Center Johannes Gutenberg, University of Mainz, Mainz, Germany

3 Instituto de Neurociencias de Castilla y León-INCyL, Universidad de Salamanca, Salamanca, Spain

4 Laboratorio de Neurobiología Comparada, Instituto Cavanilles, Universidad de Valencia, CIBERNED, Valencia, Spain

5 Departmento de Neurosciencias, Facultad de Medicina, Universidad del País Vasco, Leioa, Spain

6 Present Address: Clinical Neurobiology, German Center for Cancer Research (DKFZ), Heidelberg, Germany

7 Unidad mixta de Esclerosis múltiple y neurorregeneración, IIS Hospital La Fe, Valencia, Spain

8 Present Address: Instituto de Neurociencias, Universidad Miguel Hernández-CSIC, Alicante, Spain 